\title{
Numerical Green's function for a two-dimensional diffusion equation
}

\author{
C. A. B. Vasconcellos ${ }^{1}$, M. A. C. Ferro ${ }^{1}$, W. J. Mansur ${ }^{2}$, \\ F. S. Loureiro ${ }^{2}$ \& J. P. L. Santos ${ }^{2}$ \\ ${ }^{I}$ Department of Fortification and Construction Engineering, \\ Military Institute of Engineering, Brazil \\ ${ }^{2}$ Department of Civil Engineering, \\ COPPE/Federal University of Rio de Janeiro, Brazil
}

\begin{abstract}
This paper presents a novel form to calculate Green's function by using a numerical method. In this paper, Green's function is calculated for the twodimensional diffusion equation. The numerical Green's function is defined as Green's matrix that represents the domain of the problem to be solved in terms of the physical properties and geometrical characteristic. Green's matrix is the basis of the numerical method called 'Explicit Green's Approach' (ExGA) that allows explicit time marching with a time step larger than the one required by other methods found in the literature, without losing precision. The method uses Green's matrix which is determined numerically by the Finite Element Method (FEM). The paper presents one application in heat conduction and another in groundwater flow, demonstrating that the results are quite accurate when compared to analytical solutions and to other numerical solutions.
\end{abstract}

Keywords: Green's function, Green's matrix, ExGA, time integration, diffusion equation.

\section{Introduction}

Green's functions are very powerful tools for obtaining solutions to diffusion equation. They can also be applied to find solution of other phenomena which are described by the same type of equation, i.e., those that involve solution of diffusion-type partial differential equations. A Green's function (GF) is a basic solution of a specific differential equation with homogeneous boundary 
conditions; for transient heat conduction, a GF describes the temperature caused by an instantaneous, local energy pulse. In addition to originating efficient numerical solution procedures, GF based methods also provide a better understanding of the nature of diffusion processes for heat conduction.

Besides, the application of GFs in diffusion equation has several advantages. First, it is a powerful and flexible method, since the derived GFs for a given geometry may be used in conjunction with a variety of initial and boundary conditions; second, a systematic procedure is available for obtaining GFs, i.e., once these functions are obtained and tabulated, they may be used without any effort spent on the details of their derivation; third, 1-D GFs may be used as building blocks to obtain 2- and 3-D solutions to suitable problems. The specifics of the multiplication process to obtain 2- and 3-D solutions are presented by Özişik [1].

Finding GFs corresponding to certain physical phenomena, possibly incorporated with suitably prescribed boundary data, is one of the fundamental subjects in mathematical physics. The knowledge of GFs can serve as a basic ingredient to construct the fields via superposition under distributed sources and general boundary data.

GFs have been used in the solution of equations of transient heat conduction for many decades, a classic text on the subject is the book by Carslaw and Jaeger [2], in which an introduction to the use of GFs based methods for heat conduction problems is presented. In that study they obtained the GFs through Laplace transforms. Other important reference concerning GFs is the book of Özişik [1] where the use and advantages of GFs based methods are described.

A short review about the Green's function can be found in Mansur et al. [3]. Moreover, in this article it is presented the ExGA method for linear transient heat conduction. Before that, this method had been presented to wave propagation equation by Mansur et al. [4]. Recently, Loureiro et al. [5] presented a hybrid time/Laplace integration method based on numerical Green's functions in conduction heat transfer, that utilizes the Stehfest and the Zakian Laplace inversion schemes to compute numerically Green's functions.

The framework proposed in this article includes a novel calculating form to GF using a numerical method. The numerical GF is defined as Green's matrix, that is the basis of numerical method called 'Explicit Green's Approach' (ExGA) that allows explicit time marching with a time step larger than the one required by other methods found in the literature, without losing precision. The method uses the Green's matrix that is determined numerically by the Finite Element Method.

The paper presents one application in heat conduction and another in groundwater flow, where it is shown that the results are quite accurate when compared to analytical solutions and to other numerical solutions

In the next section, a review and the mathematical background of the GF are shown. In section 3 Green's matrix and the ExGA method are presented. In section 4, two examples are discussed. Finally, in the last section, some conclusions about this work are presented. 


\section{Analytical Green's function}

This section presents a discussion about the physical significance of the GF and a general expression for the solution of the diffusion equation with energy generation, non-homogeneous boundary conditions, and a given initial condition, in terms of the GF.

In this paper, we consider the following two-dimensional non-homogeneous initial boundary-value problem of heat conduction in homogeneous and isotropic medium:

$$
\begin{gathered}
\nabla^{2} \mathrm{~T}(\mathrm{x}, \mathrm{y}, \mathrm{t})+\frac{1}{\mathrm{k}} \mathrm{g}(\mathrm{x}, \mathrm{y}, \mathrm{t})=\frac{1}{\alpha} \frac{\partial \mathrm{T}(\mathrm{x}, \mathrm{y}, \mathrm{t})}{\partial \mathrm{t}}, \text { in } \Omega, \mathrm{t}>0, \\
\mathrm{k}_{\mathrm{i}} \frac{\partial \mathrm{T}}{\partial \mathrm{n}_{\mathrm{i}}}+\mathrm{h}_{\mathrm{i}} \mathrm{T}=\mathrm{h}_{\mathrm{i}} \mathrm{T}_{\infty \mathrm{i}} \equiv \mathrm{f}_{\mathrm{i}}(\mathrm{x}, \mathrm{y}, \mathrm{t}), \text { on } \Gamma, \mathrm{t}>0, \\
\mathrm{~T}(\mathrm{x}, \mathrm{y}, \mathrm{t})=\mathrm{T}_{0}(\mathrm{x}, \mathrm{y}), \text { in } \Omega, \mathrm{t}=0,
\end{gathered}
$$

where $\nabla^{2}$ is the two-dimensional Laplace operator, $\frac{\partial}{\partial \mathrm{n}_{\mathrm{i}}}$ denotes differentiation along the outward-draw normal to the boundary $\Gamma_{\mathrm{i}}, \mathrm{i}=1,2, \ldots, \mathrm{N} ; \mathrm{N}$ being the number of continuous boundaries in the domain. For generality it is assumed that the generation term $g(x, y, t)$ and the boundary-condition function $f_{i}(x, y, t)$ vary with both position and time; and $\alpha$ (thermal diffusivity), $\mathrm{T}_{\infty}$ (external ambient temperature), $\mathrm{k}_{\mathrm{i}}$ (thermal conductivity) and $\mathrm{h}_{\mathrm{i}}$ (heat transfer coefficient) are treated as constants.

According to Özişik [1], the GF concerning (1)-(3) can be determined by considering the following auxiliary problem for the same domain $\Omega$ :

$$
\begin{gathered}
\nabla^{2} \mathrm{G}\left(\mathrm{x}, \mathrm{y}, \mathrm{t} \mid \mathrm{x}^{\prime}, \mathrm{y}^{\prime}, \tau\right)+\frac{1}{\mathrm{k}} \delta\left(\mathrm{x}-\mathrm{x}^{\prime}\right) \delta\left(\mathrm{y}-\mathrm{y}^{\prime}\right) \delta(\mathrm{t}-\tau)=\frac{1}{\alpha} \frac{\partial \mathrm{G}}{\partial \mathrm{t}}, \mathrm{t}>\tau, \\
\mathrm{k}_{\mathrm{i}} \frac{\partial \mathrm{G}}{\partial \mathrm{n}_{\mathrm{i}}}+\mathrm{h}_{\mathrm{i}} \mathrm{G}=0, \mathrm{t}>0,
\end{gathered}
$$

Notice that the GF obeys the causality requirement which states that the GF is zero for $t<\tau$ [6]. Furthermore, the source term in (4) is a unit impulsive source for the two-dimensional problem considered here. The delta function $\delta\left(x-x^{\prime}\right) \delta\left(y-y^{\prime}\right)$ represents a line heat source located at $\left(x^{\prime}, y^{\prime}\right)$, while the delta function $\delta(t-\tau)$ indicates that it is an instantaneous heat source releasing its energy spontaneously at time $\mathrm{t}=\tau$.

GF $G\left(x, y, t \mid x^{\prime}, y^{\prime}, \tau\right)$ for the two-dimensional represents the temperature at point (x,y) in the domain $\Omega$ and at time $\mathrm{t}$ due to an instantaneous point source of 
unit strength, located at point $\left(\mathrm{x}^{\prime}, \mathrm{y}^{\prime}\right)$, releasing its energy spontaneously at time $\mathrm{t}=\tau$.

The auxiliary problem satisfied by the GF is valid over the same domain $\Omega$ as the original physical problem presented by (1), but the boundary conditions given by (5) is the homogeneous version of the boundary conditions given by (2) and the initial condition is zero.

On the basis of this definition, the physical significance of GF may be interpreted as:

$$
\mathrm{G}\left(\mathrm{x}, \mathrm{y}, \mathrm{t} \mid \mathrm{x}^{\prime}, \mathrm{y}^{\prime}, \tau\right) \equiv \mathrm{G}(\text { effect } \mid \text { impulse })
$$

\section{Numerical Green's function}

The present section describes the Explicit Green's Approach for integrating the diffusion equation. The algorithm is based on the calculation of the GF in nodal coordinates by the finite element method. The spatial discretization, using the finite element method (FEM), of the two-dimensional diffusion equation can be represented in matrix form as (Hughes [7]):

$$
[\mathrm{C}]\{\dot{\mathrm{T}}(\mathrm{t})\}+[\mathrm{K}]\{\mathrm{T}(\mathrm{t})\}=\{\mathrm{F}(\mathrm{t})\},
$$

with the initial condition,

$$
\{\mathrm{T}(0)\}=\{\mathrm{T}\}^{0},
$$

where $[\mathrm{C}]$ is the capacitance matrix, $[\mathrm{K}]$ is the thermal conductance matrix, and $\{\mathrm{F}\}$ is a vector of equivalent nodal heat loads [3].

Applying the Laplace transform to (7) (Boyce and DiPrima [8]), and following the procedure presented by Mansur et al. [3], the final expression of the vector temperature in terms of Green's matrix is:

$$
\{\mathrm{T}(\mathrm{t})\}=[\mathrm{G}(\mathrm{t})][\mathrm{C}]\{\mathrm{T}(0)\}+\int_{0}^{\mathrm{t}}[\mathrm{G}(\mathrm{t}-\tau)]\{\mathrm{F}(\tau)\} \mathrm{d} \tau .
$$

GF is usually called impulsive response of the system, as it is explained in Özişik [9]. It can be obtained numerically from (7), considering homogeneous boundary conditions and an impulsive heat source applied at a source point, which coincides with a node of the space mesh. Then, Green's matrix can be obtained by the solution of the following system of ordinary differential equations in the time:

$$
[\mathrm{C}][\dot{\mathrm{G}}(\mathrm{t})]+[\mathrm{K}][\mathrm{G}(\mathrm{t})]=[\mathrm{I}] \delta(\mathrm{t})
$$


with the initial condition,

$$
[\mathrm{G}(0)]=0 \text {, }
$$

where [I] represents the identity matrix and $\delta(t)$ is the Dirac delta function.

The problem described by (10) is equivalent to the homogeneous initial value problem with application of a certain initial condition (see Özişik [9]). In the present work, it was verified that the correct equivalence is given by the problem presented below:

$$
[\mathrm{C}][\mathrm{G}(\mathrm{t})]+[\mathrm{K}][\mathrm{G}(\mathrm{t})]=0
$$

with the initial condition,

$$
[\mathrm{G}(0)]=[\mathrm{C}]^{-1} \text {. }
$$

Here Green's matrix is computed implicitly through the range $[0, \Delta \mathrm{t}]$ with the Crank-Nicolson scheme. It is worth to point out that the new method becomes more stable and accurate when a sub-step procedure is adopted to obtain the Green's matrix. If the capacitance matrix is lumped (diagonal), computing its inverse is fairly cheap.

Assuming that the time step is $\Delta \mathrm{t}$ the temperature solution vector at any time can be evaluated recursively as:

$$
\{\mathrm{T}(\mathrm{t})\}=[\mathrm{G}]^{\Delta \mathrm{t}}[\mathrm{C}]\{\mathrm{T}\}^{\mathrm{t}}+\int_{0}^{\Delta \mathrm{t}}[\mathrm{G}(\Delta \mathrm{t}-\tau)]\{\mathrm{F}(\mathrm{t}+\tau)\} \mathrm{d} \tau .
$$

Observe that the convolution integral of the above equation can be evaluated by any integral quadrature formulae. In the present work, the convolution integral is calculated by Newton-Cotes approximations with interpolation polynomials of order one (trapezoidal rule).

\section{Numerical examples}

In this section, two examples are presented to illustrate the methodology employed in this work. The objectives of the examples are to simulate twodimensional diffusion problems with applications in heat conduction and groundwater flow. In the following analyses, the relation $(\Delta \mathrm{t})_{\mathrm{FEM}}=(\Delta \mathrm{t})_{\mathrm{ExGA}} / \mathrm{ss}$ is adopted, so that ss stands for the number of sub-steps employed to compute the Green's matrix.

\section{Example 1}

In the first example a thin rectangular plate of sides $\mathrm{a}=10.0 \mathrm{~m}$ and $\mathrm{b}=8.0 \mathrm{~m}$ was considered. The adopted physical coefficients were $\alpha=1.0 \mathrm{~m} / \mathrm{s}$, $\mathrm{k}=1.0 \mathrm{~W} /{ }^{\circ} \mathrm{C} . \mathrm{m}$. The Green's matrix was determined by the FEM using 80 and 
320 square elements for the spatial discretization and the finite difference method with Crank-Nicolson scheme for time integration. The ExGA method was compared to the FEM with the same spatial discretization and with the same implicit scheme in the time discretization used in the calculus of the Green's matrix, i.e., FEM-CN.

The initial condition is $\mathrm{T}(\mathrm{x}, \mathrm{y}, 0)=0.0^{\circ} \mathrm{C}$ over the entire domain. The boundaries condition are: $\mathrm{q}(\mathrm{x}, 0, \mathrm{t})=0.0 \mathrm{~W} / \mathrm{m}, \mathrm{q}(0, \mathrm{y}, \mathrm{t})=10.0 \mathrm{~W} / \mathrm{m}, \mathrm{T}(\mathrm{x}, \mathrm{b}, \mathrm{t})=$ $300.0^{\circ} \mathrm{C}$ and $\mathrm{T}(\mathrm{a}, \mathrm{y}, \mathrm{t})=0.0^{\circ} \mathrm{C}$, as shown in fig. 1 below.

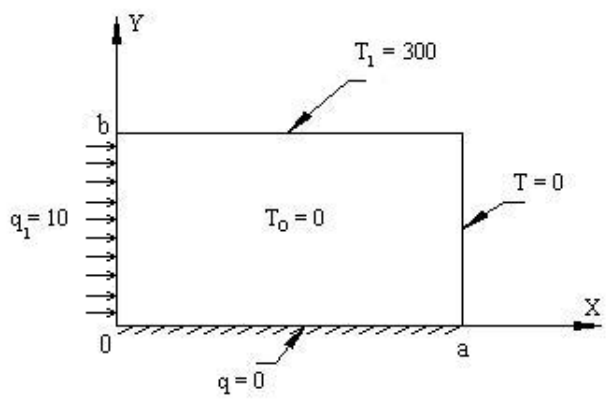

Figure 1: Domain, initial condition and boundaries conditions for example 1.

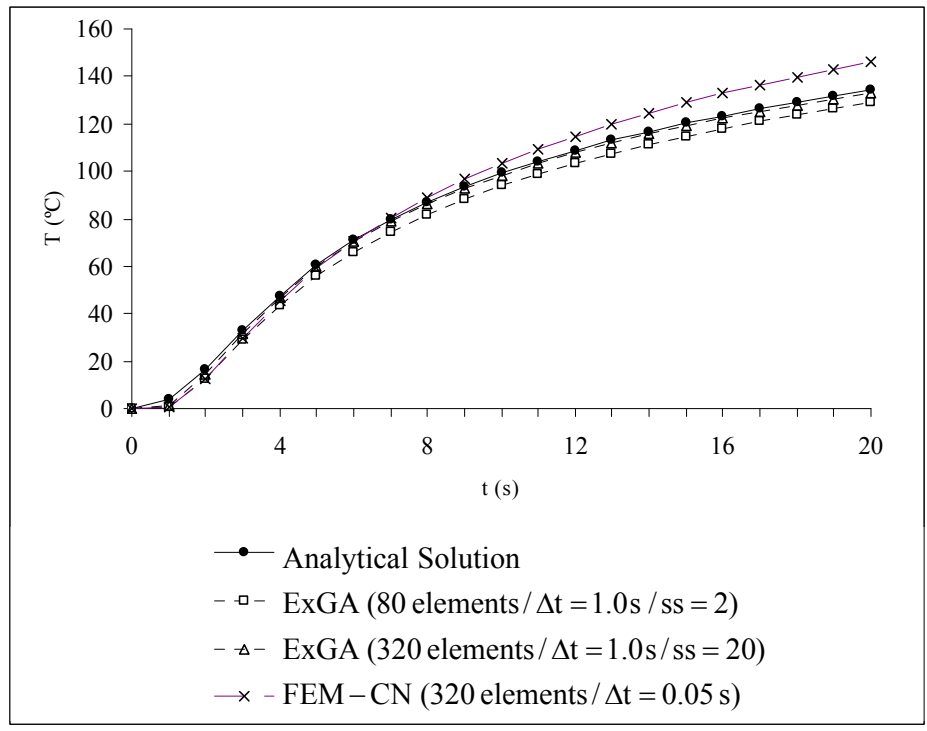

Figure 2: $\quad$ Temperature at the central point of domain of example 1.

Note that the boundary conditions are different on each side of the domain, which makes convergence more difficult. However, the ExGA method converges to the analytical solution when the mesh is refined from 80 to 320 elements. The 
time step used by ExGA method was $\Delta \mathrm{t}=1.0 \mathrm{~s}$ with 2 and 20 sub-steps to calculate Green's matrix, while $\Delta \mathrm{t}=0.05 \mathrm{~s}$ was used by FEM-CN. The results are shown in fig. 2, where the ExGA method is compared with the analytical solution presented by Beck et al [10].

\section{Example 2 - Theis problem}

Consider a transient well drawdown problem. The well is discharging at a constant rate $\mathrm{Q}$ from an extensive confined aquifer where the potentiometric surface is initially horizontal, its hydraulic head being equal to $h_{0}$. This problem was solved analytically by Theis in 1935 [11] and the drawdown at a radius $r$ from the well is shown in the classical book by Wang and Anderson [12].

The horizontal confined aquifer of this example, whose area is $2000 \mathrm{x}$ $2000 \mathrm{~m}^{2}$, has the following physical properties: $\mathrm{T}=300 \mathrm{~m}^{2} /$ day (transmissivity) and $\mathrm{S}=0.002$ (storage coefficient). The well is discharging at a constant flow equal to $2000 \mathrm{~m}^{3} /$ day and their area of influence is marked in a tone of gray in fig.3. The initial condition of the problem is $h_{0}(x, y, 0)=10.0 \mathrm{~m}$, and the boundaries conditions are zero flow on all sides of the aquifer.

Wang and Anderson [12] presented numerical solutions for this example using FDM and FEM methods, with the same spatial discretization $(\Delta x=\Delta y=$ $100.0 \mathrm{~m}$ ) and the Crank-Nicolson scheme to carry out time marching with time step equal to $\Delta \mathrm{t}=0.01$ day.

In order to maintain the same conditions for comparison, the same mesh discretization was used for the ExGA method. Two simulations were carried out. In the first simulation we used the time step $\Delta \mathrm{t}=5.0$ days and 10 sub-steps in the calculation of the Green's matrix. These results are compared with the analytical Theis solution as depicted in fig. 4 for points located $100 \mathrm{~m}$ from the well.

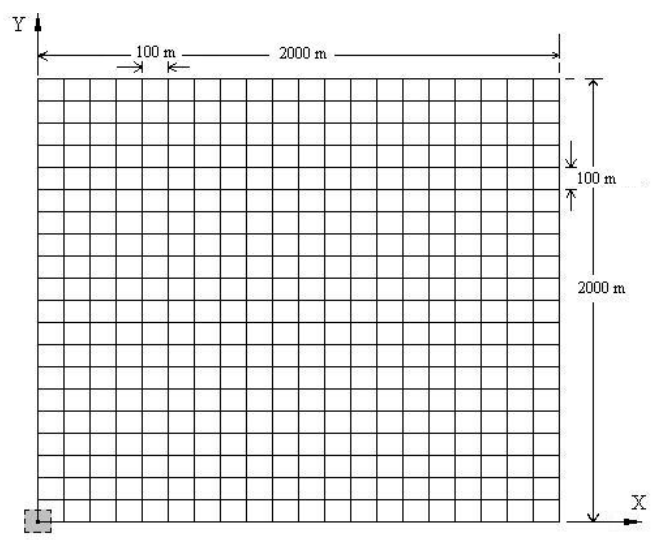

Figure 3: $\quad$ Confined aquifer - domain and spatial discretization.

In the second simulation, the final time of simulation is set to be equal to $t=$ 13.12 days. Time steps $\Delta \mathrm{t}=0.01$ days were used in the FDM-CN and FEM-CN 


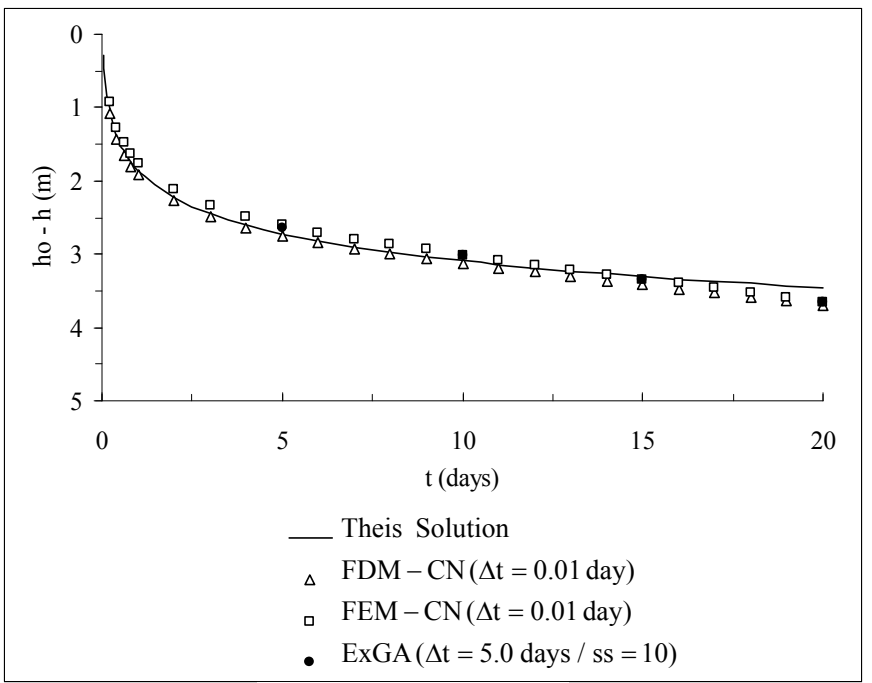

Figure 4: $\quad$ The time-drawdown curve to $100 \mathrm{~m}$ from the well.

Table 1: $\quad$ Results for $\mathrm{r}=100 \mathrm{~m}$ and $\mathrm{t}=13.12$ days.

\begin{tabular}{|c|c|c|c|}
\hline Method & $\Delta \mathrm{t}$ (days) & $\mathrm{h}_{0}-\mathrm{h}(\mathrm{m})$ & Relative errors $(\%)$ \\
\hline FDM-CN & 0.01 & 3.2582 & 0.80 \\
\hline FEM-CN & 0.01 & 3.2170 & 0.47 \\
\hline ExGA & 13.12 & 3.2798 & 1.47 \\
\hline ExGA & 1.312 & 3.2169 & 0.47 \\
\hline
\end{tabular}

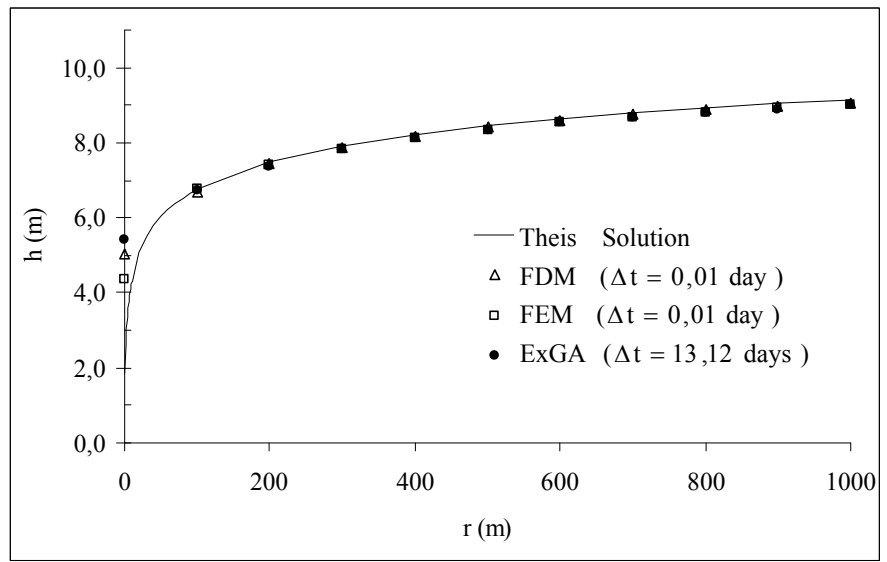

Figure 5: Hydraulic head versus distance of the well for $\mathrm{t}=13.12$ days. 
methods, while time steps equal to $\Delta \mathrm{t}=13.12$ days (with 20 sub-steps) and $\Delta \mathrm{t}=$ 1.312 days (with 10 sub-steps) were used in the ExGA method.

Table 1 presents the results for the distance $\mathrm{r}=100 \mathrm{~m}$ from the well, and their relative errors, considering the Theis Solution as reference, which in this case is equal to $3.2322 \mathrm{~m}$. Fig. 5 shows, for $\mathrm{t}=13.12$ days, the cone of depression formed by the lowering of water level near the well due to the constant flow pump.

\section{Conclusions}

The results of the examples display the expected behavior of a robust numerical method. In all the studied cases, the ExGA method provided accurate results and converged to the correct answer with refinements that do not demand great computational efforts.

This work presented a new approach for the solution of diffusion equation with application in heat conduction and groundwater flow.

Note that the inverse of the capacitance matrix is an equivalent initial condition to the unitary pulse given by the Dirac delta function. If this were not possible, less accurate numerical Green's function would be obtained, and the ExGA method would not be competitive with the classic ones.

In the ExGA method, the temperature field is calculated explicitly on time through the Green's matrix which is determined by FEM using an implicit algorithm. The use of this implicit algorithm with sub-steps to compute Green's matrix increases the computational cost; however, this apparent disadvantage becomes a positive aspect since the unconditional stability property inherent to implicit methods, such as the Crank-Nicolson scheme, is transmitted through the Green's matrix to the ExGA method.

Thus, with sub-steps not too small, one obtains an explicit algorithm which for any practical purpose can be considered unconditionally stable. In the example 1, a time step 20 times bigger than that of the FEM was considered. Already in example 2 it becomes 1000 times bigger than these. In fact using substeps allow the time-step 'length' to be as large as one wishes, the only limitation being a good representation of the time response picture.

Finally, the present paper has presented that the ExGA method can be very suitable to multiple cases where only the load conditions (boundary or source) change while domain and physical properties remain unchanged, as in this case is possible to use the same Green's matrix for all analyses.

\section{References}

[1] Özişik, M. N. Heat Conduction. Wiley, New York, 1980.

[2] Carslaw, H.S. and Jaeger, J.C. Conduction of Heat in Solids, 2nd edition, Oxford University Press, 1959.

[3] Mansur, W. J., Vasconcellos, C.A.B., Zambrozuski, N.J.M. \& Rotunno Filho, O.C., Numerical solution for the linear transient heat conduction 
equation using an explicit Green's Approach. International Journal of Heat and Mass Transfer, 52, pp. 694-701, 2009.

[4] Mansur, W. J., Loureiro, F. S., Soares Jr. D. \& Dors, C. Explicit timedomain approaches based on numerical Green's functions computed by finite differences - The ExGA family. Journal of Computational Physics, 227(1), pp. 851-870, 2007.

[5] Loureiro, F.S., Mansur, W. J. \& Vasconcellos, C.A.B, A hybrid time/Laplace integration method based on numerical Green's functions in conduction heat transfer. Computational Methods in Applied Mechanical and Engineering, accepted in 20 March 2009.

[6] Morse, P. M. \& Feshbach, H. Methods of Theoretical Physics, volumes I and II. McGraw-Hill, New York, 1st edition, 1953.

[7] Hughes, T.J.R. The Finite Element Method, Linear Static and Dynamic Finite Element Analysis. Prentice-Hall, Englewood Cliffs, New Jersey, 1987.

[8] Boyce, W.E. \& DiPrima, R.C. Elementary Differential Equation and Boundary Value Problems. John Willey and Sons, Inc., 8th edition, 2005.

[9] Özişik, M. N. Heat Conduction. John Wiley \& Sons, Inc., New York, 2nd edition, 1993.

[10] Beck, J.V., Cole, K.D., Haji-Sheikh, A. \& Litkouhi, B. Heat conduction using Green's function. Hemisphere Press. Washington, DC, 1992.

[11] Freeze, R.A. \& Cherry, J.A., Groundwater, Ed. Prentice-Hall, Inc., New Jersey, USA, 1979.

[12] Wang, H.F. \& Anderson, M.P., Introduction to Groundwater Modeling: Finite Difference and Finite Element Methods. Academic Press, Inc. San Diego, 1982. 